\title{
Minimum Phase Property of Chebyshev-Sharpened Cosine Filters
}

\author{
Miriam Guadalupe Cruz Jiménez, ${ }^{1}$ David Ernesto Troncoso Romero, ${ }^{2}$ \\ and Gordana Jovanovic Dolecek ${ }^{1}$ \\ ${ }^{1}$ Department of Electronics, INAOE, 72840 Tonantzintla, PUE, Mexico \\ ${ }^{2}$ Cátedras-CONACYT, ESCOM-IPN, 07738 Mexico City, DF, Mexico \\ Correspondence should be addressed to Miriam Guadalupe Cruz Jiménez; miriam.gcj@gmail.com
}

Received 27 May 2014; Accepted 12 January 2015

Academic Editor: Changchun Hua

Copyright (C) 2015 Miriam Guadalupe Cruz Jiménez et al. This is an open access article distributed under the Creative Commons Attribution License, which permits unrestricted use, distribution, and reproduction in any medium, provided the original work is properly cited.

\begin{abstract}
We prove that the Chebyshev sharpening technique, recently introduced in literature, provides filters with a Minimum Phase (MP) characteristic when it is applied to cosine filters. Additionally, we demonstrate that cascaded expanded Chebyshev-Sharpened Cosine Filters (CSCFs) are also MP filters, and we show that they achieve a lower group delay for similar magnitude characteristics in comparison with traditional cascaded expanded cosine filters. The importance of the characteristics of cascaded expanded CSCFs is also elaborated. The developed examples show improvements in the group delay ranged from $23 \%$ to $47 \%$ at the cost of a slight increase of usage of hardware resources. For an application of a low-delay decimation filter, the proposed scheme exhibits a $24 \%$ lower group delay, with 35\% less computational complexity (estimated in Additions per Output Sample) and slightly less usage of hardware elements.
\end{abstract}

\section{Introduction}

A Minimum Phase (MP) digital filter has all zeros on or inside the unit circle [1]. We consider in this paper MP Finite Impulse Response (FIR) filters, which find applications in cases where a long delay, usually introduced by Linear Phase (LP) FIR filters, is not allowed. Examples of these cases include data communication systems or speech and audio processing systems [2-4].

The basic building block analyzed in this paper, the cosine filter, is a simple FIR filter whose transfer function and frequency response are, respectively, given by

$$
\begin{aligned}
H(z) & =\frac{1}{2}\left[1+z^{-1}\right], \\
H\left(e^{j \omega}\right) & =\left[\cos \left(\frac{\omega}{2}\right)\right] e^{-j \omega / 2} .
\end{aligned}
$$

This filter is of special interest because of the following main reasons:

(a) It has MP property because its zero lies on the unit circle.

(b) It has a low computational complexity because it does not require multipliers, which are the most costly and power-consuming elements in a digital filter [5].

(c) It has a low usage of hardware elements, which can be translated into a low demand of chip area for implementation.

The fact that a cosine filter has the Minimum Phase characteristic becomes significant because these basic building blocks can be used to design filters with a low delay and simultaneously a low computational complexity and a low usage of hardware resources.

Due to the aforementioned characteristics, cascaded expanded cosine filters were investigated in [6]. A cascaded 
expanded cosine filter is defined as a filter with transfer function and frequency response, respectively, given by

$$
\begin{aligned}
H_{A}(z) & =\left[\prod_{k=1}^{K} H\left(z^{k}\right)\right]^{L}, \\
H_{A}\left(e^{j \omega}\right) & =\left[\prod_{k=1}^{K}\left[\cos \left(k \cdot \frac{\omega}{2}\right)\right]^{L}\right] e^{-j \omega L \sum_{k=1}^{K} k / 2},
\end{aligned}
$$

with $H(z)$ being the cosine filter given in (1), whereas $K$ and $L$ are arbitrary integers. In method [6], Rouche's theorem was employed to demonstrate that when cascaded expanded cosine filters are sharpened with a modified version of the technique from [7] (originally devised for LP FIR filters), the result is an overall FIR filter that has all its zeros on or inside the unit circle; that is, it satisfies the MP characteristic. Nevertheless, the resulting filter still has a high group delay because a large number of cascaded expanded cosine filters are needed to meet a given attenuation specification, as we see in the examples from [6].

On the other hand, the Chebyshev sharpening technique was recently introduced in [8] to design LP FIR filters based on comb subfilters for decimation applications. In that method the sharpening is performed with an $N$ th degree Chebyshev polynomial of first kind, defined as

$$
P(x)=\sum_{n=0}^{N} c_{n} \cdot x^{n}
$$

where $c_{n}$ are integers [9]. When applied to comb filters, Chebyshev sharpening provides solutions with advantages like a simple and elegant design method, a low-complexity resulting LP FIR filter, and improved attenuation characteristics in the resulting filter. However, filters from [8] are not guaranteed to have MP characteristic.

From the aforementioned literature we can extract the following observations:

(a) In MP FIR filters the reduction of the group delay is a priority.

(b) The use of cosine filters results in low-complexity multiplierless MP FIR filters.

(c) The recent Chebyshev sharpening method from [8] can improve the attenuation of cosine filters and is a potentially useful approach to preserve a simple multiplierless solution with a lower group delay in comparison with simple cascaded expanded cosine filters.

Motivated by the remarks listed above, we present in this paper the following contributions:

(1) The mathematical proof that the use of Chebyshev sharpening in cosine filters, which produces Chebyshev-Sharpened Cosine Filters (CSCFs), guarantees resulting multiplierless filters with all of their zeros placed on the unit circle, that is, with MP property: this demonstration hinges upon the factorization of the transfer function of the CSCF into secondorder sections, taking advantage of the antisymmetry of the roots of the Chebyshev polynomial.
(2) The mathematical proof that cascaded expanded CSCFs have also all of their zeros placed on the unit circle: this demonstration is a simple extension of aforementioned proof for CSCFs.

(3) The explanation of how cascaded expanded CSCFs can be efficiently employed in the design of MP FIR filters.

(4) Examples where it is shown that cascaded expanded CSCFs are useful to obtain the same stopband attenuation as cascaded expanded cosine filters but with a lower group delay: from these examples we see an improvement from $23 \%$ to $47 \%$ in the reduction of the group delay, at the cost of a slight increase of the usage of hardware resources. For an application in a decimation filter embedded into a low-delay oversampled Analog-to-Digital Converter (ADC), the proposed scheme exhibits a $24 \%$ lower group delay referred to high rate, with $35 \%$ less computational complexity (estimated in Additions per Output Sample) and slightly less usage of hardware elements.

Following this introduction, Section 2 presents the definition of CSCFs and cascaded expanded CSCFs. The proofs of MP characteristic in CSCFs and cascaded expanded CSCFs are given in Sections 3 and 4, respectively. In Section 5 we provide details on the characteristics and applications of the cascaded expanded CSCFs. Then, Section 6 presents examples and discussion of results. Finally, concluding remarks are given in Section 7.

\section{Definition of Chebyshev-Sharpened Cosine Filter (CSCF) and Cascaded Expanded CSCF}

We define the transfer function and the frequency response of an $\mathrm{N}$ th order Chebyshev-Sharpened Cosine Filter (CSCF), respectively, as

$$
\begin{gathered}
H_{C, N}(z, \gamma)=\sum_{n=0}^{N} z^{-(N-n) / 2} \cdot c_{n} \cdot[\gamma H(z)]^{n}, \\
H_{C, N}\left(e^{j \omega}, \gamma\right)=\left[\sum_{n=0}^{N} c_{n} \cdot\left[\gamma \cos \left(\frac{\omega}{2}\right)\right]^{n}\right] e^{-j \omega N / 2},
\end{gathered}
$$

with

$$
\gamma \leq \frac{1}{\cos (\pi / 2-\pi / 4 R)}
$$

where $c_{n}$ are the coefficients of the Chebyshev polynomial of first kind, represented in (5), and $H(z)$ is given in (1). To obtain a low-complexity multiplierless implementation, the constant $\gamma$ must be expressible as a Sum of Powers of Two (SOPOT). To this end, we set

$$
\gamma=f\left(2^{-B}\left\lfloor\frac{2^{B}}{\cos (\pi / 2-\pi / 4 R)}\right\rfloor, 1\right),
$$

where $f(a, b)$ denotes "the closest value less than or equal to $a$ that can be realized with at most $b$ adders" and $\lfloor x\rfloor$ denotes 
rounding $x$ to the closest integer less than or equal to $x$. To provide an improved attenuation around the zero of the cosine filter, $\gamma$ must be as close as possible to its upper limit [8]. This is achieved by increasing the integer $B$. The value $R$ in (8)-(9) is usually set as an integer equal to or greater than 2 for applications in decimation processes [8]. However, we will allow for more flexibility to the parameter $R$ in this paper, as will be explained in the next section.

The transfer function and frequency response of a cascaded expanded CSCF are, respectively, defined as

$$
\begin{aligned}
& G(z)=\prod_{m=1}^{M}\left[H_{C, N_{m}}\left(z^{m}, \gamma_{m}\right)\right]^{K_{m}}, \\
& G\left(e^{j \omega}\right)=\left\{\prod_{m=1}^{M}\left[\sum_{n=0}^{N_{m}} c_{n} \cdot\left[\gamma_{m} \cos \left(m \cdot \frac{\omega}{2}\right)\right]^{n}\right]^{K_{m}}\right\} \\
& \cdot e^{-j \omega \sum_{m=1}^{M} m \cdot K_{m} \cdot N_{m} / 2},
\end{aligned}
$$

where the integer $M$ indicates the number of cascaded CSCF blocks, each of them repeated $K_{m}$ times, with $m=$ $1,2, \ldots, M$. Every value of $m$ is a distinct factor that expands a different CSCF whose corresponding order is $N_{m}$. These CSCFs have different factors $\gamma_{m}$, which can be obtained using (9), just replacing $B$ by $B_{m}$ and $R$ by $R_{m}$, where $B_{m}$ and $R_{m}$ are integer parameters that correspond to the $m$ th CSCF in the cascade. Figure 1(a) shows the structure of the CSCF, where we have that $d_{i}=c_{2 i+v}$, with $i=0,1,2, \ldots, D=(N-v) / 2$ and with $v=1$ if $N$ is odd or $v=0$ if $N$ is even. Dashed blocks in Figure 1(a) appear only if $N$ is odd. Figure 1(b) presents the structure of the cascaded expanded CSCF whose transfer function is given in (10).

\section{Proof of Minimum Phase Property in CSCFs}

The proof starts with the expression of the Chebyshev polynomial from (5) in the form of a product of first-order terms as [9]

$$
\begin{aligned}
P(x) & =\sum_{n=0}^{N} c_{n} \cdot x^{n}=\prod_{n=1}^{N}\left(x-\sigma_{n}\right), \\
\sigma_{n} & =\cos \left(\frac{\pi}{2} \cdot \frac{2 n-1}{N}\right) .
\end{aligned}
$$

On the other hand, we rewrite the transfer function of the CSCF from (6) as

$$
H_{C, N}(z, \gamma)=z^{-N / 2} \sum_{n=0}^{N} c_{n} \cdot\left[z^{1 / 2} \gamma H(z)\right]^{n} .
$$

Using (12), and after simple rearrangement of terms, we express $H_{C, N}(z, \gamma)$ as follows:

$$
H_{C, N}(z, \gamma)=\prod_{n=1}^{N}\left[\gamma H(z)-z^{-1 / 2} \sigma_{n}\right],
$$

which can be rewritten as

$$
H_{C, N}(z, \gamma)= \begin{cases}\prod_{n=1}^{N / 2}\left\{\left[\gamma H(z)-z^{-1 / 2} \sigma_{n}\right]\left[\gamma H(z)-z^{-1 / 2} \sigma_{N-(n-1)}\right]\right\} ; & N \text { even, } \\ {\left[\gamma H(z)-z^{-1 / 2} \sigma_{\lceil N / 2\rceil}\right] \prod_{n=1}^{\lceil N / 2\rceil-1}\left\{\left[\gamma H(z)-z^{-1 / 2} \sigma_{n}\right]\left[\gamma H(z)-z^{-1 / 2} \sigma_{N-(n-1)}\right]\right\} ;} & N \text { odd }\end{cases}
$$

where $\lceil x\rceil$ denotes rounding $x$ to the closest integer greater than or equal to $x$.

At this point, it is worth highlighting that the antisymmetry relations

$$
\begin{aligned}
\sigma_{n} & =-\sigma_{N-(n-1)}, \quad n=1,2, \ldots,\left\lceil\frac{N}{2}\right\rceil, \\
\sigma_{\lceil N / 2\rceil} & =0 \quad \text { for } N \text { odd }
\end{aligned}
$$

hold [9]. Thus, replacing (17) in (16), and after simple manipulation of terms, we have

$$
\begin{aligned}
H_{C, N}(z, \gamma) & = \begin{cases}\prod_{n=1}^{N / 2} Q_{n}(z) ; & N \text { even, } \\
\gamma H(z) \prod_{n=1}^{\lceil N / 2\rceil-1} Q_{n}(z) ; & N \text { odd },\end{cases} \\
Q_{n}(z) & =\gamma^{2} H^{2}(z)-\sigma_{n}^{2} z^{-1} .
\end{aligned}
$$

From (18) we have that $H_{C, N}(z, \gamma)$ consists of a product of either several terms $Q_{n}(z)$ if $N$ is even or several terms $Q_{n}(z)$ and a term $\gamma H(z)$ if $N$ is odd, with $n=1,2, \ldots, N$. Thus, to prove the MP property of the CSCF, it is only necessary to ensure that $Q_{n}(z)$ and $\gamma H(z)$ have MP characteristic for all values $n$.

Using (1), it is easy to see that the term $\gamma H(z)$ has a root on the unit circle and thus it corresponds to a MP filter. On the other hand, replacing (1) into (19) and after simple rearrangement of terms, we get

$$
Q_{n}(z)=\frac{\gamma^{2}}{4}\left[1-\left(\frac{4 \sigma_{n}^{2}}{\gamma^{2}}-2\right) z^{-1}+z^{-2}\right] .
$$

From (20) it is easy to show that the roots of $Q_{n}(z)$ are placed on the unit circle; that is,

$$
\begin{aligned}
Q_{n}(z) & =\left(1-e^{j 2 \varphi_{n}} z^{-1}\right)\left(1-e^{-j 2 \varphi_{n}} z^{-1}\right), \\
\varphi_{n} & =\arccos \left(\sigma_{n} \cdot \gamma^{-1}\right),
\end{aligned}
$$




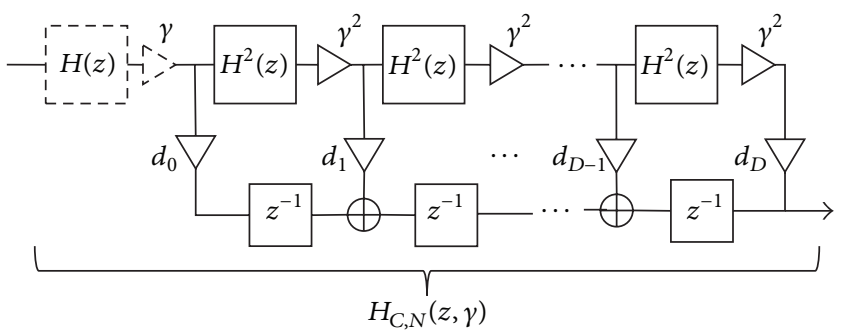

(a)

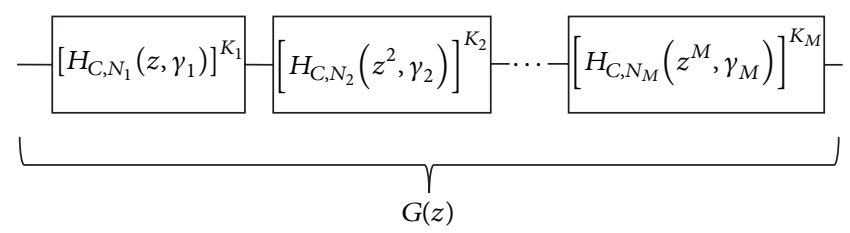

(b)

Figure 1: General structure of the filters: (a) Chebyshev-Sharpened Cosine Filter (CSCF); (b) cascaded expanded CSCF.
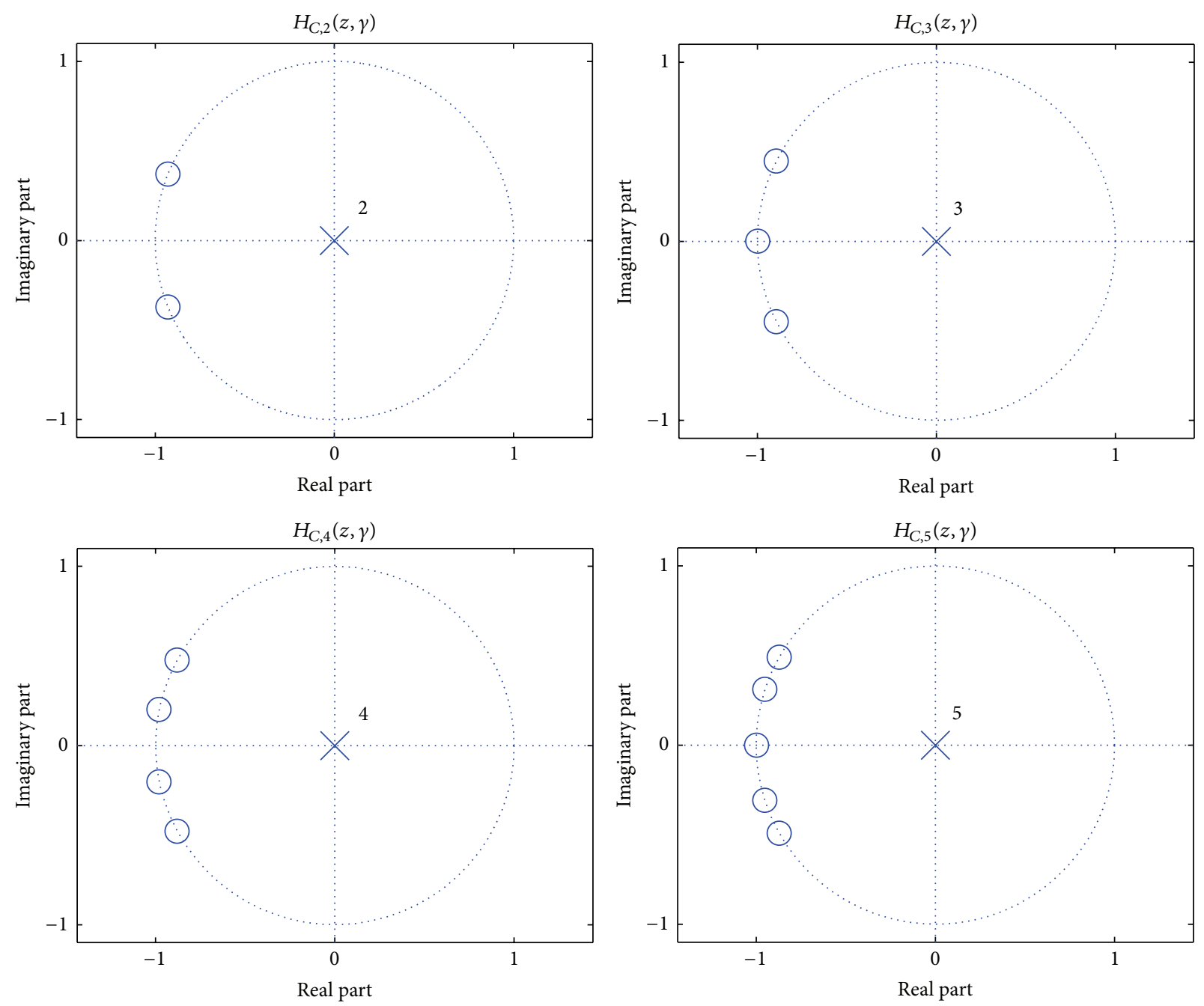

Figure 2: Pole-zero plots for CSCFs $H_{C, 2}(z, \gamma), H_{C, 3}(z, \gamma), H_{C, 4}(z, \gamma)$, and $H_{C, 5}(z, \gamma)$, where $\gamma=2^{-3} \times 15$.

if the argument $\sigma_{n} \cdot \gamma^{-1}$ in (22) is preserved into the range $[-1,1]$. From (13) we have that $-1 \leq \sigma_{n} \leq 1$ holds. Additionally, by setting

$$
R \geq 0.5
$$

in (8)-(9), we ensure $\gamma \geq 1$. Under this condition for $R$, we have that $-1 \leq \gamma^{-1} \leq 1$ holds. In this case, $Q_{n}(z)$ has its roots on the unit circle for all the valid values $n$ and, as a consequence, the filter $H_{C, N}(z, \gamma)$ has a MP characteristic.

Figure 2 shows the pole-zero plots for the filters $H_{C, 2}(z, \gamma), H_{C, 3}(z, \gamma), H_{C, 4}(z, \gamma)$, and $H_{C, 5}(z, \gamma)$. For all of these filters, we have $\gamma=2^{-3} \times 15$, which is implemented with just one subtraction. 


\section{Proof of Minimum Phase Property in Cascaded Expanded CSCFs}

The proof starts with the expression of every CSCF of the cascaded expanded CSCFs from (10) in the form of a product of second-order expanded transfer functions using (18) and (20); that is,

$$
\begin{aligned}
& H_{C, N_{m}}\left(z^{m}, \gamma_{m}\right) \\
& = \begin{cases}\prod_{n=1}^{N_{m} / 2} Q_{n}\left(z^{m}\right) ; & N_{m} \text { even, } \\
\gamma_{m} H\left(z^{m}\right) \prod_{n=1}^{\left\lceil N_{m} / 2\right\rceil-1} Q_{n}\left(z^{m}\right) ; & N_{m} \text { odd, }\end{cases} \\
& Q_{n}\left(z^{m}\right)=\frac{\gamma_{m}^{2}}{4}\left[1-\left(\frac{4 \sigma_{n}^{2}}{\gamma_{m}^{2}}-2\right) z^{-m}+z^{-2 m}\right],
\end{aligned}
$$

where $m=1,2, \ldots, M$ and $n=1,2, \ldots, N_{m}$. Since the transfer function of the cascaded expanded CSCF from (10) consists of a product of several terms $\left[H_{C, N_{m}}\left(z^{m}, \gamma_{m}\right)\right]^{K_{m}}$ with different values $m$, it is only necessary to ensure that $H_{C, N_{m}}\left(z^{m}, \gamma_{m}\right)$ has a MP characteristic for all values $m$. Moreover, from (24) we see that $H_{C, N_{m}}\left(z^{m}, \gamma_{m}\right)$ is expressed as a product of either several terms $Q_{n}\left(z^{m}\right)$ if $N_{m}$ is even or several terms $Q_{n}\left(z^{m}\right)$ and $\gamma_{m} H\left(z^{m}\right)$ if $N_{m}$ is odd. Thus, to prove the MP property in cascaded expanded CSCFs we only need to ensure that $Q_{n}\left(z^{m}\right)$ and $\gamma_{m} H\left(z^{m}\right)$ have MP characteristic for all values $n$ and $m$.

By replacing (1) in the term $\gamma_{m} H\left(z^{m}\right)$ and then making the resulting expression equal to zero, we can find the $m$ roots of $\gamma_{m} H\left(z^{m}\right)$. These roots turn out to be the $m$ complex roots of -1 , which have unitary magnitude. Thus, $\gamma_{m} H\left(z^{m}\right)$ has MP characteristic, since its roots are placed on the unit circle. On the other hand, using (21) we can express (25) as follows:

$$
\begin{aligned}
Q_{n}\left(z^{m}\right) & =\left(1-e^{j 2 \varphi_{n}} z^{-m}\right)\left(1-e^{-j 2 \varphi_{n}} z^{-m}\right), \\
\varphi_{n} & =\arccos \left(\sigma_{n} \cdot \gamma_{m}^{-1}\right) .
\end{aligned}
$$

To preserve the argument $\sigma_{n} \cdot \gamma_{m}^{-1}$ in $(27)$ into the range $[-1,1]$, we set

$$
R_{m} \geq 0.5, \quad m=1,2, \ldots, M
$$

Under this condition for $R_{m}$, we have that $-1 \leq \gamma_{m}^{-1} \leq 1$ holds. In this case, the respective $m$ roots of factors $\left(1-e^{j 2 \varphi_{n}} z^{-m}\right)$ and $\left(1-e^{-j 2 \varphi_{n}} z^{-m}\right)$ in (25) are the $m$ roots of the complex numbers $e^{j 2 \varphi_{n}}$ and $e^{-j 2 \varphi_{n}}$, which have unitary magnitude for all the valid values $n$. Therefore, $Q_{n}\left(z^{m}\right)$ has MP characteristic, since its roots are placed on the unit circle. Finally, since $Q_{n}\left(z^{m}\right)$ and $\gamma_{m} H\left(z^{m}\right)$ have MP characteristic, the overall cascaded expanded CSCF from (9), $G(z)$, also has MP characteristic.

Figure 3 shows the pole-zero plots for the filters $H_{C, 2}\left(z^{5}, \gamma\right), H_{C, 3}\left(z^{4}, \gamma\right), H_{C, 4}\left(z^{3}, \gamma\right)$, and $H_{C, 5}\left(z^{2}, \gamma\right)$. For all these filters, we have $\gamma=2^{-3} \times 15$, which is implemented with just one subtraction.

\section{Characteristics and Applications of Cascaded Expanded CSCFs}

A cascaded expanded CSCF has both MP and LP characteristics. The former was proven in Section 4, whereas the latter is easily seen from the frequency response $G\left(e^{j \omega}\right)$ given in (11). A consequence of this is that the cascaded expanded CSCF has a passband droop in its magnitude response. Due to this passband droop, the cascaded expanded CSCF should be employed only to provide a given attenuation requirement of an overall MP FIR filter over a prescribed stopband region (depending on the application). Thus, the resulting structure to design an overall MP FIR filter can be associated with the prefilter-equalizer scheme of [10], shown in Figure 4, where the prefilter provides the required attenuation whereas the equalizer corrects the passband droop of the prefilter. The cascaded expanded CSCF, with transfer function $G(z)$ defined in (10), can be used as prefilter. Note that since a cascaded expanded cosine filter (whose transfer function $H_{A}(z)$ is defined in (3)) also has both LP and MP properties, it is used as prefilter in [6].

Even though this paper is not focused on the design of the equalizer, it is worthwhile to spend some words on how this filter could be designed. An Infinite Impulse Response (IIR) filter with optimally located poles based, for example, in the Least Squares criterion as shown in [11] can form a proper equalizer. However, FIR filters are usually preferred over their IIR counterparts because they have guaranteed stability, they are free of limit-cycle oscillations, and their polyphase decomposition in multirate schemes allows them to reduce the computational load, among other characteristics [12]. Thus, we are more concerned here with FIR equalizers. Since a FIR equalizer with LP characteristic has its zeros placed in quadruplets around the unit circle [1], it does not accomplish the MP characteristic. Therefore, a MP FIR equalizer (i.e., that filter whose zeros appear inside the unit circle) does not have a Linear Phase.

When a LP FIR filter is designed by sharpening cascaded expanded cosine filters with the traditional sharpening polynomial $3 x^{2}-2 x^{3}$ from [7], the resulting filter has a prefilter given by $\left[H_{A}(z)\right]^{2}$ and a LP equalizer given by $\left[3 z^{-D}-2 H_{A}(z)\right]$, where $D$ is the group delay of $H_{A}(z)$ used to preserve the Linear Phase characteristic. In method [6] the delay $D$ has been removed to obtain a MP FIR equalizer. Thus, a first option would be to use the same approach of [6] to design a FIR equalizer. However, it is worth highlighting that the recent LP droop compensators proposed in literature (see, e.g., $[13,14]$ ) are novel low-complexity alternatives to the aforementioned LP equalizer based on the traditional sharpening. Inspired by these alternatives, a more convenient approach would be to design MP droop compensators as counterparts of the MP equalizer based on sharpening, proposed in [6].

Besides method [6], other design methods for MP FIR filters have been introduced, for example, in [15-22]. They can be classified in general terms as methods based on cepstrum [15-17] and methods based on the design of a LP FIR filter [18-22]. However, in general, these methods have the inconvenience of producing filtering solutions that require 

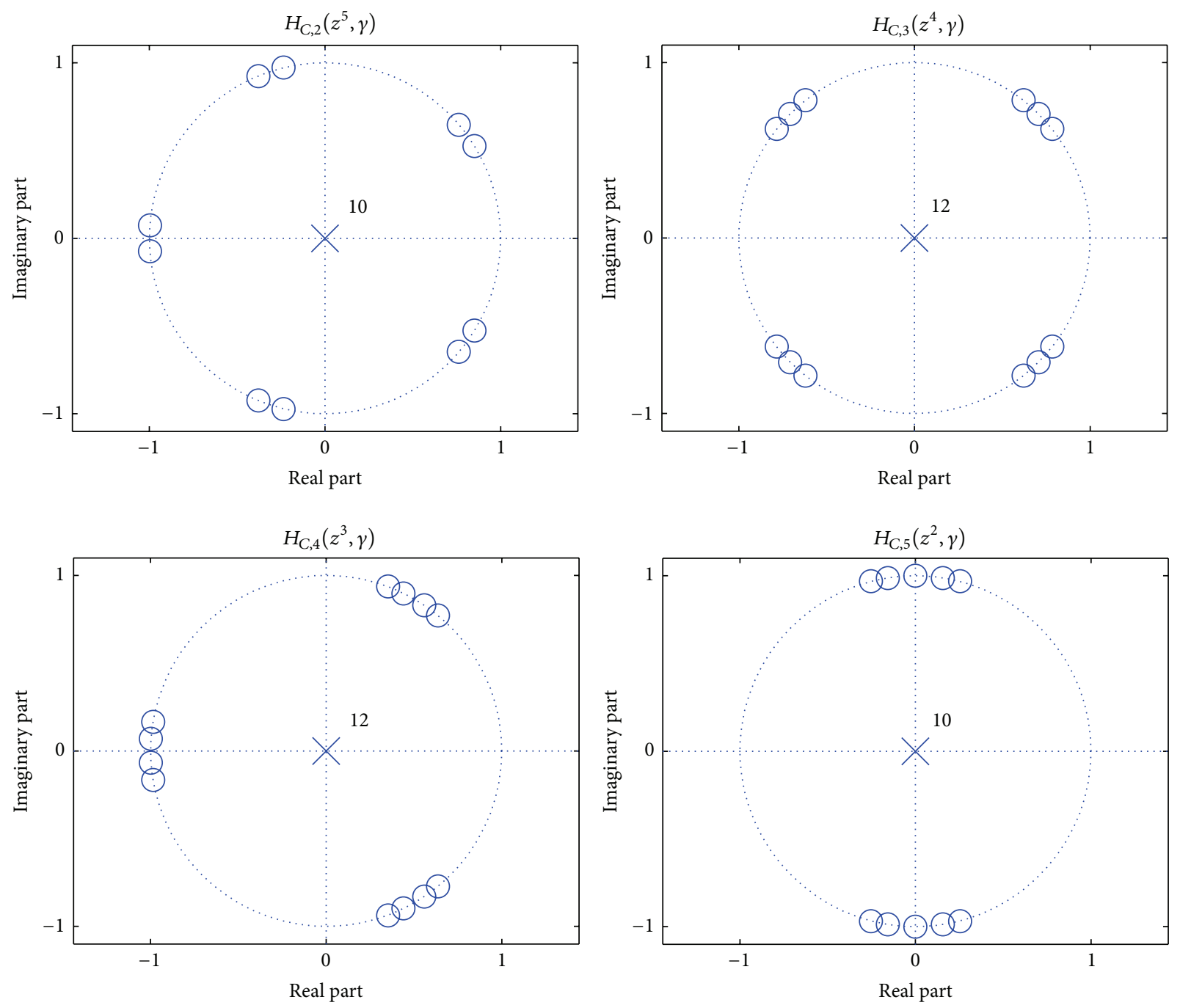

FIgURe 3: Pole-zero plots for cascaded expanded CSCFs $H_{C, 2}\left(z^{5}, \gamma\right), H_{C, 3}\left(z^{4}, \gamma\right), H_{C, 4}\left(z^{3}, \gamma\right)$, and $H_{C, 5}\left(z^{2}, \gamma\right)$, where $\gamma=2^{-3} \times 15$.

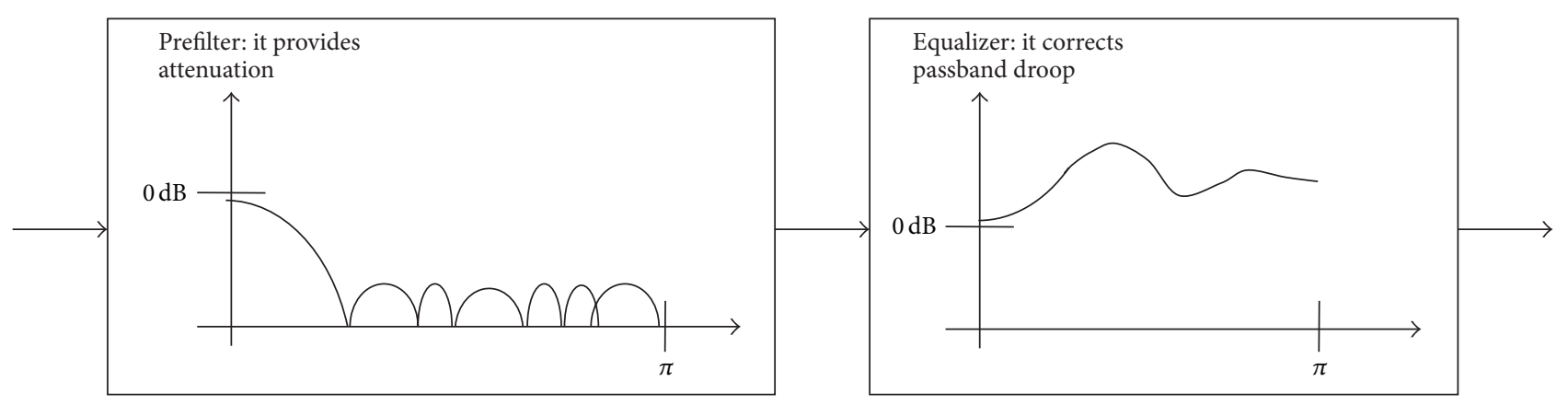

FIGURE 4: Prefilter-equalizer scheme to design an overall FIR filter. For a MP FIR filter design, the prefilter and the equalizer must be MP filters.

multipliers, which are the most costly elements in a digital filter [5]. To solve this problem, the cascaded expanded CSCF can be used as a prefilter to implement an overall MP FIR filter using several multiplierless CSCFs. A similar approach can be followed with the use of a cascaded expanded cosine filter from [6]. Nevertheless, as we mentioned earlier, the problem with method [6] is that the resulting filter requires a large cascade of expanded cosine filters, increasing the group delay of the resulting filter.

Finally, it is worth highlighting that, in comparison to the filter $H_{A}(z)$, the filter $G(z)$ has many more parameters, namely, $N_{m}, K_{m}, B_{m}$, and $R_{m}$, with $m=1,2, \ldots, M$, 
TABLE 1: First half of the symmetric impulse response of the filter designed with method [6] in Example 1.

\begin{tabular}{lccccc}
\hline$n$ & $h_{A}(n)$ & $n$ & $h_{A}(n)$ & $n$ & $h_{A}(n)$ \\
\hline 1 & 0.000030517578125 & 9 & 0.004943847656250 & 17 & 0.037902832031250 \\
2 & 0.000091552734375 & 10 & 0.007110595703125 & 18 & 0.043304443359375 \\
3 & 0.000183105468750 & 11 & 0.009887695312500 & 19 & 0.048431396484375 \\
4 & 0.000396728515625 & 12 & 0.013275146484375 & 20 & 0.052825927734375 \\
5 & 0.000732421875000 & 13 & 0.017272949218750 & 21 & 0.056488037109375 \\
6 & 0.001281738281250 & 14 & 0.021881103515625 & 22 & 0.058959960937500 \\
7 & 0.002136230468750 & 15 & 0.026916503906250 & 23 & 0.060241699218750 \\
8 & 0.003295898437500 & 16 & 0.032409667968750 & & \\
\hline
\end{tabular}

TABLE 2: First half of the symmetric impulse response of the proposed filter in Example 1.

\begin{tabular}{lccccc}
\hline$n$ & $g(n)$ & $n$ & $g(n)$ & $n$ & $g(n)$ \\
\hline 1 & 0.000365884150812 & 7 & 0.014876445801089 & 13 & 0.055883940227800 \\
2 & 0.001024551768820 & 8 & 0.020473561194634 & 14 & 0.061849547506437 \\
3 & 0.002122204221257 & 9 & 0.027011012207314 & 15 & 0.066392852921837 \\
4 & 0.003834694340150 & 10 & 0.034112182959393 & 16 & 0.069389704077153 \\
5 & 0.006627799290290 & 11 & 0.041585018019638 & 17 & 0.070269686319927 \\
6 & 0.010243501009105 & 12 & 0.049072257144308 & & \\
\hline
\end{tabular}

to be tuned in order to find a desired attenuation. This characteristic provides more flexibility for the design of MP FIR filters in comparison to $H_{A}(z)$. Moreover, by setting $M=$ $K, N_{m}=1, R_{m}=0.5$, and $K_{m}=L$ for all $m$ in (10), we obtain the same expression as (3). Thus, the cascaded expanded CSCF from (10) is a generalized case of (3).

\section{Examples and Discussion}

This section presents a couple of examples (Examples 1 and 2) that compare the cascaded expanded CSCFs $G(z)$ with cascaded expanded cosine filters $H_{A}(z)$ from [6]. This comparison is made in terms of

(a) group delay, measured in samples and defined as follows [1]:

$$
\tau(\omega)=-\frac{d}{d \omega}\left\{\arg \left[F\left(e^{j \omega}\right)\right]\right\},
$$

where $F\left(e^{j \omega}\right)$ is the frequency response of the corresponding filter;

(b) implementation complexity, measured in the required number of adders and delays for a given attenuation over a prescribed stopband region.

Additionally, an engineering application is provided in Example 3, namely, the antialiasing filtering process used in the first stage of a two-stage decimation structure applied in a low-delay Sigma-Delta ADC for audio systems, detailed in [3]. In this case, comparisons are made in terms of group delay referred to high rate, computational complexity counted in Additions per Output Sample (APOS), and number of hardware elements assuming that both filters, the one used in method [3] and the proposed filter, are implemented in recursive form.
Example 1. Design a MP FIR filter with minimum attenuation equal to $60 \mathrm{~dB}$ over the range from $\omega=0.17 \pi$ to $\omega=\pi$ (see Figure 1 of [6]).

In [6], the filter employed to accomplish such characteristic is obtained from (3) using $K=5$ and $L=3$. The group delay is obtained by replacing these values in (4) and then using (4) in (29). This filter requires 15 adders and 45 delays, but it has a group delay of 22.5 samples.

If we use $M=4, N_{1}=N_{3}=N_{4}=3, N_{2}=4, R_{1}=3, R_{2}=$ $1.5, R_{3}=0.9$, and $R_{4}=2$, with $B_{m}=4$ and $K_{m}=1$ for all $m$ in (10), we get a filter whose group delay, obtained by replacing the aforementioned parameters in (11) and then using (11) in (29), is 16 samples, that is, nearly $30 \%$ less delay than that of [6]. Since this filter uses 30 adders and 44 delays, the price to pay is $100 \times\{[(30+44) /(15+45)]-1\} \approx 23 \%$ of additional implementation complexity. Figure 5 shows the magnitude responses and group delays of both filters. Moreover, Tables 1 and 2 present, respectively, the first half of the symmetric impulse response of the filter designed with method [6] and the proposed filter.

Example 2. Design a MP FIR filter with minimum attenuation equal to $100 \mathrm{~dB}$ over the range from $\omega=0.15 \pi$ to $\omega=\pi$ (see Example 1 of [6]).

In [6], the filter employed to accomplish such characteristic is obtained from (3) using $K=7$ and $L=4$. The group delay is obtained by replacing these values in (4) and then using (4) in (29). This filter requires 24 adders and 120 delays. However, its group delay is 56 samples.

By using $M=4, N_{1}=4, N_{2}=6, N_{3}=4, N_{4}=8, R_{1}=6$, $R_{2}=2, R_{3}=0.8, R_{4}=1.2, B_{1}=2, B_{2}=B_{4}=3, B_{3}=4$, and $K_{m}=$ 1 for all $m$ in (10), we get a filter whose group delay, obtained by replacing the aforementioned parameters in (11) and then 


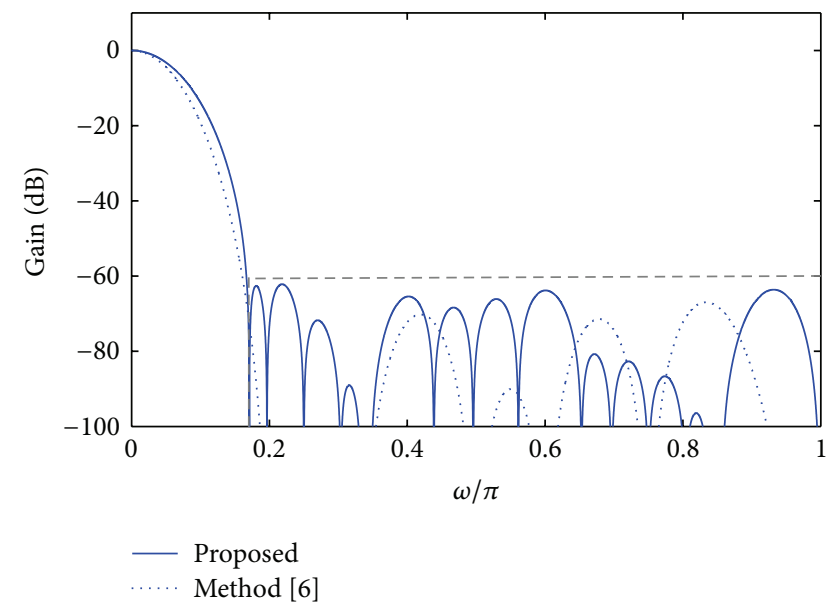

(a)

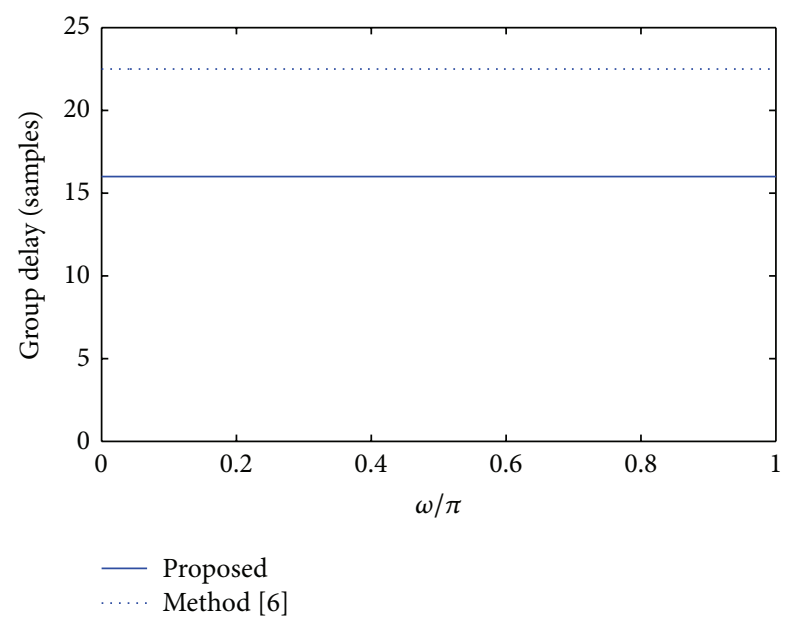

(b)

Figure 5: Magnitude responses (a) and group delays (b) of the cascaded expanded CSCF (10) and the cascaded expanded cosine filter from [6] (3), accomplishing the attenuation required in Example 1.

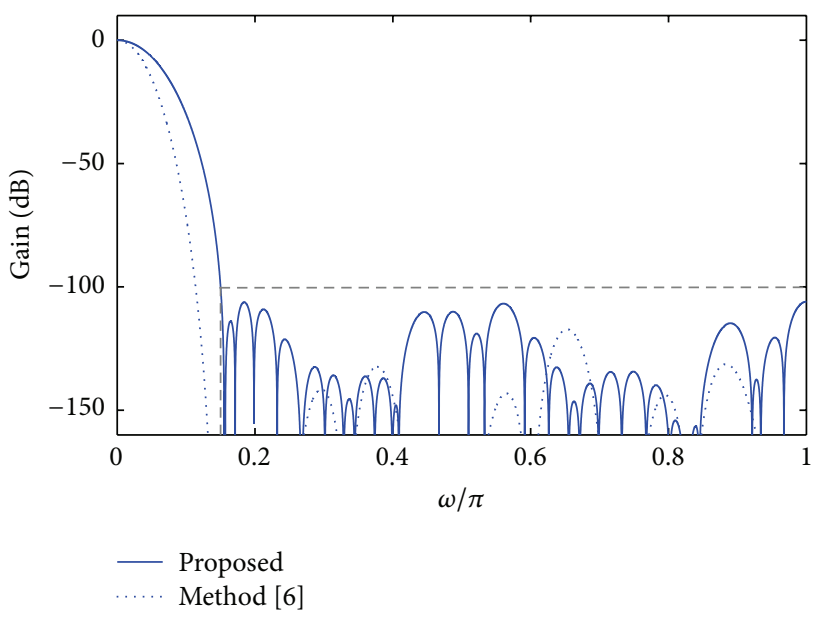

(a)

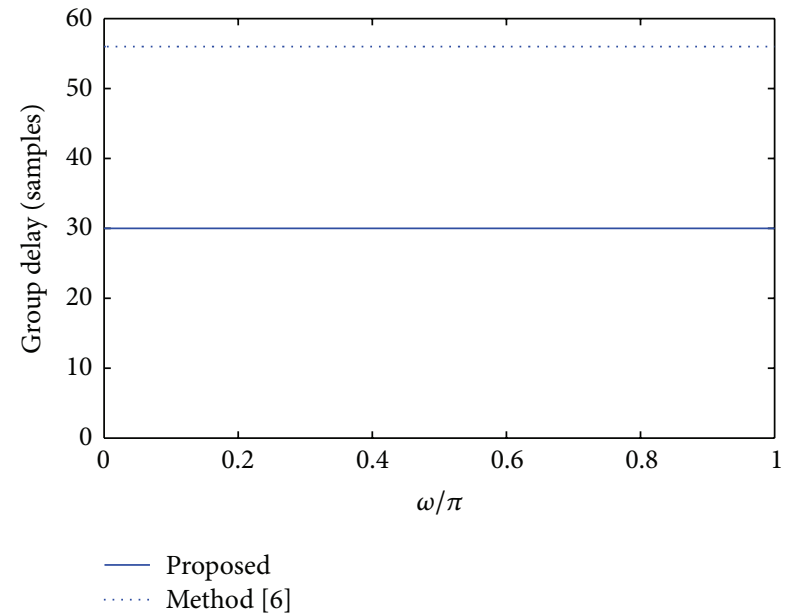

(b)

Figure 6: Magnitude responses (a) and group delays (b) of the cascaded expanded CSCF (10) and the cascaded expanded cosine filter from [6] (3), accomplishing the attenuation required in Example 2.

using (11) in (29), is 30 samples, that is, approximately $47 \%$ less delay than that of [6]. This filter uses 58 adders and 90 delays; thus the price to pay is a $100 \times\{[(58+90) /(24+$ $120)]-1\} \approx 3 \%$ of additional implementation complexity. Figure 6 shows the magnitude responses and group delays of both filters. Tables 3 and 4 , respectively, show the first half of the symmetric impulse response of the filter of method [6] and the proposed filter in Example 2.

Table 5 summarizes the results from the previous examples. From them we observe that the cascaded expanded CSCFs achieve a lower group delay in comparison to the cascaded expanded cosine filters from [6]. This characteristic, desirable for MP filters, occurs because CSCFs take advantage of the zero-rotation effect (see Figures 2 and 3), provided by Chebyshev sharpening, to achieve a given attenuation using less cascaded filters. Nevertheless, since every CSCF needs in general a few more hardware resources (adders and delays) than their cascaded cosine counterparts, the price to pay is an increase in the implementation complexity. Note, however, that the resulting filters still are low-complexity multiplierless solutions.

In the following example we show the design of the antialiasing filter used in the first stage of a two-stage decimation structure applied in a low-delay Sigma-Delta ADC for audio systems, where the second stage is a FIR filter with droop compensation characteristic followed by a downsampler by $2[3]$.

Example 3. Design a MP FIR filter for a decimation factor $M=32$ and residual decimation factor $R=2$, with minimum 
TABLE 3: First half of the symmetric impulse response of the filter of method [6] in Example 2.

\begin{tabular}{lccccc}
\hline$n$ & $h_{A}(n)$ & $n$ & $h_{A}(n)$ & $n$ & $h_{A}(n)$ \\
\hline 1 & 0.000000003725290 & 20 & 0.000197932124138 & 39 & 0.010904885828495 \\
2 & 0.000000014901161 & 21 & 0.000268835574389 & 40 & 0.012328892946243 \\
3 & 0.000000037252903 & 22 & 0.000360459089279 & 41 & 0.013833809643984 \\
4 & 0.000000089406967 & 23 & 0.0004777448105812 & 42 & 0.015406861901283 \\
5 & 0.000000189989805 & 24 & 0.000624939799309 & 43 & 0.017032675445080 \\
6 & 0.000000372529030 & 25 & 0.000808782875538 & 44 & 0.018693551421165 \\
7 & 0.000000707805157 & 26 & 0.001035392284393 & 45 & 0.020369183272123 \\
8 & 0.000001281499863 & 27 & 0.001311570405960 & 46 & 0.022037297487259 \\
9 & 0.000002223998308 & 28 & 0.001644581556320 & 47 & 0.023674391210079 \\
10 & 0.000003740191460 & 29 & 0.002041984349489 & 48 & 0.025255739688873 \\
11 & 0.000006116926670 & 30 & 0.002511218190193 & 49 & 0.026756063103676 \\
12 & 0.000009730458260 & 31 & 0.003059625625610 & 50 & 0.028150811791420 \\
13 & 0.000015132129192 & 32 & 0.003694280982018 & 51 & 0.029415860772133 \\
14 & 0.000023052096367 & 33 & 0.004421260207891 & 52 & 0.030528664588928 \\
15 & 0.000034391880035 & 34 & 0.005245819687843 & 53 & 0.031469188630581 \\
16 & 0.000050395727158 & 35 & 0.006171941757202 & 54 & 0.032219946384430 \\
17 & 0.000072613358498 & 36 & 0.007201746106148 & 55 & 0.032766595482826 \\
18 & 0.000102937221527 & 37 & 0.008335486054420 & 56 & 0.033098891377449 \\
19 & 0.000143751502037 & 38 & 0.009571358561516 & 57 & 0.033210486173630 \\
\hline
\end{tabular}

TABLE 4: First half of the symmetric impulse response of the proposed filter in Example 2.

\begin{tabular}{lccccc}
\hline$n$ & $g(n)$ & $n$ & $g(n)$ & $n$ & $g(n)$ \\
\hline 1 & 0.000001616556925 & 12 & 0.002625166590998 & 23 & 24 \\
2 & 0.000006351272540 & 13 & 0.003671733802111 & 25 & 0.030805600150354 \\
3 & 0.000017617899954 & 14 & 0.004998834324957 & 26 & 0.037993214348607 \\
4 & 0.000040689528112 & 15 & 0.006638831718593 & 0.041220432511362 \\
5 & 0.000085616315587 & 16 & 0.008615391803219 & 27 & 0.044049047537715 \\
6 & 0.000163942825303 & 17 & 0.010940958165390 & 28 & 0.046372196978920 \\
7 & 0.000292679744504 & 18 & 0.013612477204781 & 39 & 0.048100695572177 \\
8 & 0.00049325324069 & 19 & 0.016611117272267 & 30 & 0.049166485033492 \\
9 & 0.000793906372572 & 20 & 0.019897013838157 & 31 & 0.049526751322073 \\
10 & 0.001225195293310 & 21 & 0.023412420954797 & & \\
11 & 0.001822819899364 & 22 & 0.027079443485406 & & \\
\hline
\end{tabular}

TABLE 5: Comparison of results in Examples 1 and 2.

\begin{tabular}{|c|c|c|c|c|}
\hline & \multicolumn{2}{|c|}{ Example 1} & \multicolumn{2}{|c|}{ Example 2} \\
\hline & Proposed & Method [6] & Proposed & Method [6] \\
\hline Group delay (samples) & 16 & 22.5 & 30 & 56 \\
\hline $\begin{array}{l}\text { Complexity of implementation } \\
\text { (number of adders/number of delays) }\end{array}$ & $30 / 44$ & $15 / 45$ & $58 / 90$ & $24 / 120$ \\
\hline $\begin{array}{l}\text { \% improvement in group delay } \\
\text { (compared with method [6]) }\end{array}$ & $\approx 30 \%$ & - & $\approx 47 \%$ & - \\
\hline $\begin{array}{l}\text { \% increase in complexity of implementation } \\
\text { (compared with method [6]) }\end{array}$ & $\approx 23 \%$ & - & $\approx 3 \%$ & - \\
\hline
\end{tabular}

attenuation equal to $95 \mathrm{~dB}$ over the range of frequencies from $\omega_{1, k}$ to $\omega_{2, k}$, where these frequencies are given by

$$
\begin{array}{ll}
\omega_{1, k}=\frac{\pi}{16} k-\frac{\pi}{64}, & \text { for } k=1,2, \ldots, 16, \\
\omega_{2, k}= \begin{cases}\frac{\pi}{16} k+\frac{\pi}{64} ; & \text { for } k=1,2, \ldots, 15, \\
\pi ; & \text { for } k=16 .\end{cases}
\end{array}
$$

In [3], the filter employed to accomplish such characteristic is obtained from method [23]. Its transfer function $H_{\text {comb }}(z)$ and frequency response $H_{\text {comb }}\left(e^{j \omega}\right)$ are, respectively, given as

$$
\begin{gathered}
H_{\text {comb }}(z)=\left[\frac{1-z^{-32}}{1-z^{-1}}\right]^{10}=\left[\sum_{k=0}^{31} z^{-k}\right]^{10}, \\
H_{\text {comb }}\left(e^{j \omega}\right)=\left[\frac{\sin (16 \omega)}{\sin (\omega / 2)}\right]^{10} e^{-j \omega(10 \times 31) / 2} .
\end{gathered}
$$




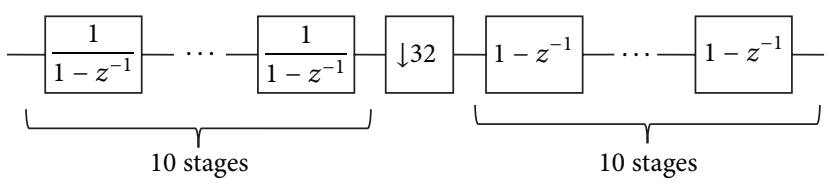

FIGURE 7: CIC structure for the first-stage decimation filter of Example 3, used in method [3].

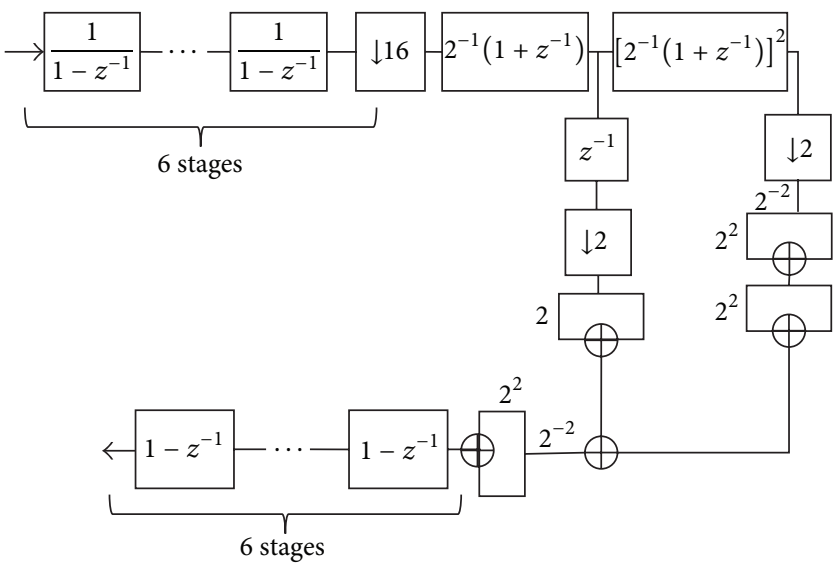

Figure 8: Proposed CIC-based structure for the first-stage decimation filter of Example 3.

This filter has a group delay of $(10 \times 31) / 2=155$ samples. Moreover, its Cascaded Integrator-Comb (CIC) structure, shown in Figure 7, performs $10 \times 32+10=330$ Additions per Output Sample (APOS) and uses 20 adders and 20 delays.

Now consider the proposed filter, whose transfer function $H_{\text {prop }}(z)$ is given by

$$
H_{\text {prop }}(z)=\left[\frac{1-z^{-32}}{1-z^{-1}}\right]^{6} G(z)=\left[\sum_{k=0}^{31} z^{-k}\right]^{6} G(z),
$$

where $G(z)$ is an expanded CSCF given by

$$
G(z)=\left[H_{C, 3}\left(z^{16}, \gamma\right)\right], \quad \gamma=2^{-2} \times 5
$$

The frequency response $H_{\text {prop }}\left(e^{j \omega}\right)$ is given by

$$
\begin{aligned}
H_{\text {prop }}\left(e^{j \omega}\right)= & {\left[\frac{\sin (16 \omega)}{\sin (\omega / 2)}\right]^{6} } \\
& \cdot\left\{\sum_{n=0}^{3} c_{n} \cdot\left[\gamma \cos \left(16 \cdot \frac{\omega}{2}\right)\right]^{n}\right\} \\
& \cdot e^{-j \omega[(6 \times 31) / 2+8 \times 3]} .
\end{aligned}
$$

This filter has a group delay of $[(6 \times 31) / 2+8 \times 3]=117$ samples, which is approximately $24 \%$ less delay than that of [3]. The filter $G(z)$ can be moved after a downsampling by 16 because it is actually a CSCF expanded by 16 . Thus, the resulting CIC-based structure, shown in Figure 8, performs $(6 \times 32+6)+(3 \times 2+5+6)=215$ Additions per Output Sample (APOS), that is, nearly $35 \%$ less computational complexity with regard to the filter used in [3]. Moreover, this filter uses 20 adders and 16 delays, which represents $10 \%$ lower usage of hardware resources compared with method [3].

Figure 9 shows the magnitude responses and group delays referred to high rate of both the filter used in method [3] and the proposed filter. Tables 6 and 7 show the first half of the symmetric impulse response referred to high rate of the filters obtained with method [3] and the proposed method, respectively, whereas the summary of results is given in Table 8.

It is worth highlighting that the implementation of the comb decimation filter in a CIC structure has been employed in method [3] due to its regularity and simplicity, which has a low usage of hardware resources (see Figure 7). However, the price to pay for such desirable characteristics is a high computational complexity. Our proposed solution has taken advantage of the possibility of factorize the decimation factor $M=32$ as $M=16 \times 2$. With this we have used an expanded-by16 CSCF as an additional filter that contributes to improving the attenuation in the first stopband, where the comb filter has the worst attenuation. The first advantage of doing so is observed in the reduction of the number of Integrator-Comb stages from 10 to 6 . Moreover, since the CSCF can operate at a sampling rate reduced by 16 , the computational complexity of the decimation process is reduced and the number of hardware elements is also reduced. Of course one can resort to other types of architectures, such as the nonrecursive form of the comb filter and its subsequent polyphase decomposition. However, this decreases the computational complexity at the cost of a considerable increase of the number of hardware elements. 
TABLE 6: First half of the symmetric impulse response of the filter of method [3], referred to high rate, in Example 3.

\begin{tabular}{|c|c|c|c|c|c|}
\hline$n$ & $h_{\text {comb }}(n)$ & $n$ & $h_{\text {comb }}(n)$ & $n$ & $h_{\mathrm{comb}}(n)$ \\
\hline 1 & 0.000000000000001 & 53 & 0.000015313628992 & 105 & 0.003067434040211 \\
\hline 2 & 0.000000000000009 & 54 & 0.000017891039557 & 106 & 0.003254277926132 \\
\hline 3 & 0.000000000000049 & 55 & 0.000020842073902 & 107 & 0.003447970844412 \\
\hline 4 & 0.000000000000195 & 56 & 0.000024211829441 & 108 & 0.003648431922869 \\
\hline 5 & 0.000000000000635 & 57 & 0.000028049632732 & 109 & 0.003855552947449 \\
\hline 6 & 0.000000000001778 & 58 & 0.000032409292032 & 110 & 0.004069197518604 \\
\hline 7 & 0.000000000004445 & 59 & 0.000037349349356 & 111 & 0.004289200321715 \\
\hline 8 & 0.000000000010161 & 60 & 0.000042933330064 & 112 & 0.004515366520934 \\
\hline 9 & 0.000000000021592 & 61 & 0.000049229987809 & 113 & 0.004747471285302 \\
\hline 10 & 0.000000000043183 & 62 & 0.000056313542416 & 114 & 0.004985259455488 \\
\hline 11 & 0.000000000082048 & 63 & 0.000064263908115 & 115 & 0.005228445358849 \\
\hline 12 & 0.000000000149178 & 64 & 0.000073166909260 & 116 & 0.005476712779839 \\
\hline 13 & 0.000000000261062 & 65 & 0.000083114480507 & 117 & 0.005729715091984 \\
\hline 14 & 0.000000000441798 & 66 & 0.000094204848246 & 118 & 0.005987075556853 \\
\hline 15 & 0.000000000725811 & 67 & 0.000106542689959 & 119 & 0.006248387794461 \\
\hline 16 & 0.000000001161297 & 68 & 0.000120239268071 & 120 & 0.006513216428630 \\
\hline 17 & 0.000000001814526 & 69 & 0.000135412534785 & 121 & 0.006781097909681 \\
\hline 18 & 0.000000002775158 & 70 & 0.000152187204385 & 122 & 0.007051541515750 \\
\hline 19 & 0.000000004162737 & 71 & 0.000170694789450 & 123 & 0.007324030532772 \\
\hline 20 & 0.000000006134560 & 72 & 0.000191073597477 & 124 & 0.007598023611877 \\
\hline 21 & 0.000000008895111 & 73 & 0.000213468684471 & 125 & 0.007872956301611 \\
\hline 22 & 0.000000012707302 & 74 & 0.000238031762123 & 126 & 0.008148242750906 \\
\hline 23 & 0.000000017905744 & 75 & 0.000264921055368 & 127 & 0.008423277577236 \\
\hline 24 & 0.000000024912339 & 76 & 0.000294301107232 & 128 & 0.008697437892806 \\
\hline 25 & 0.000000034254466 & 77 & 0.000326342528076 & 129 & 0.008970085480113 \\
\hline 26 & 0.000000046586074 & 78 & 0.000361221686589 & 130 & 0.009240569106890 \\
\hline 27 & 0.000000062712022 & 79 & 0.000399120340084 & 131 & 0.009508226969148 \\
\hline 28 & 0.000000083616030 & 80 & 0.000440225201999 & 132 & 0.009772389249925 \\
\hline 29 & 0.000000110492611 & 81 & 0.000484727444753 & 133 & 0.010032380780283 \\
\hline 30 & 0.000000144783421 & 82 & 0.000532822136527 & 134 & 0.010287523788204 \\
\hline 31 & 0.000000188218447 & 83 & 0.000584707610845 & 135 & 0.010537140720208 \\
\hline 32 & 0.000000242862512 & 84 & 0.000640584768306 & 136 & 0.010780557119830 \\
\hline 33 & 0.000000311167585 & 85 & 0.000700656310233 & 137 & 0.011017104546500 \\
\hline 34 & 0.000000396031394 & 86 & 0.000765125904472 & 138 & 0.011246123517914 \\
\hline 35 & 0.000000500862858 & 87 & 0.000834197284103 & 139 & 0.011466966458595 \\
\hline 36 & 0.000000629654824 & 88 & 0.000908073280357 & 140 & 0.011679000637130 \\
\hline 37 & 0.000000787064622 & 89 & 0.000986954791600 & 141 & 0.011881611074397 \\
\hline 38 & 0.000000978502888 & 90 & 0.001071039690856 & 142 & 0.012074203405099 \\
\hline 39 & 0.000001210231111 & 91 & 0.001160521674976 & 143 & 0.012256206674994 \\
\hline 40 & 0.000001489468318 & 92 & 0.001255589059213 & 144 & 0.012427076056412 \\
\hline 41 & 0.000001824507242 & 93 & 0.001356423521699 & 145 & 0.012586295464958 \\
\hline 42 & 0.000002224840312 & 94 & 0.001463198803009 & 146 & 0.012733380060741 \\
\hline 43 & 0.000002701295694 & 95 & 0.001576079366785 & 147 & 0.012867878617966 \\
\hline 44 & 0.000003266183590 & 96 & 0.001695219028193 & 148 & 0.012989375747416 \\
\hline 45 & 0.000003933452897 & 97 & 0.001820759557685 & 149 & 0.013097493957082 \\
\hline 46 & 0.000004718858246 & 98 & 0.001952829268212 & 150 & 0.013191895537066 \\
\hline 47 & 0.000005640137379 & 99 & 0.002091541594584 & 151 & 0.013272284255899 \\
\hline 48 & 0.000006717198673 & 100 & 0.002236993674174 & 152 & 0.013338406856462 \\
\hline 49 & 0.000007972318561 & 101 & 0.002389264938603 & 153 & 0.013390054340938 \\
\hline 50 & 0.000009430348458 & 102 & 0.002548415726373 & 154 & 0.013427063035536 \\
\hline 51 & 0.000011118930675 & 103 & 0.002714485926685 & 155 & 0.013449315427145 \\
\hline 52 & 0.000013068722691 & 104 & 0.002887493664900 & 156 & 0.013456740765633 \\
\hline
\end{tabular}


TABLE 7: First half of the symmetric impulse response of the proposed filter, referred to high rate, in Example 3.

\begin{tabular}{|c|c|c|c|c|c|}
\hline$n$ & $h_{\text {prop }}(n)$ & $n$ & $h_{\text {prop }}(n)$ & $n$ & $h_{\text {prop }}(n)$ \\
\hline 1 & 0.000000000132290 & 41 & 0.000200623662253 & 81 & 0.005950250387700 \\
\hline 2 & 0.000000000793741 & 42 & 0.000227922965264 & 82 & 0.006248059555550 \\
\hline 3 & 0.000000002778093 & 43 & 0.000258185937789 & 83 & 0.006551124134355 \\
\hline 4 & 0.000000007408248 & 44 & 0.000291642986915 & 84 & 0.006858858787878 \\
\hline 5 & 0.000000016668557 & 45 & 0.000328534155745 & 85 & 0.007170639614659 \\
\hline 6 & 0.000000033337115 & 46 & 0.000369109128687 & 86 & 0.007485806476325 \\
\hline 7 & 0.000000061118044 & 47 & 0.000413627236743 & 87 & 0.007803665325892 \\
\hline 8 & 0.000000104773790 & 48 & 0.000462357462807 & 88 & 0.008123490536077 \\
\hline 9 & 0.000000170257408 & 49 & 0.000515576579015 & 89 & 0.008444527227601 \\
\hline 10 & 0.000000264844857 & 50 & 0.000573567284102 & 90 & 0.008765993597494 \\
\hline 11 & 0.000000397267286 & 51 & 0.000636616340754 & 91 & 0.009087083247406 \\
\hline 12 & 0.000000577843325 & 52 & 0.000705012712966 & 92 & 0.009406967511909 \\
\hline 13 & 0.000000818611377 & 53 & 0.000779045703398 & 93 & 0.009724797786806 \\
\hline 14 & 0.000001133461906 & 54 & 0.000859003090723 & 94 & 0.010039707857438 \\
\hline 15 & 0.000001538269730 & 55 & 0.000945169266991 & 95 & 0.010350816226988 \\
\hline 16 & 0.000002051026306 & 56 & 0.001037823374976 & 96 & 0.010657228444788 \\
\hline 17 & 0.000002692305398 & 57 & 0.001137237445536 & 97 & 0.010958041789391 \\
\hline 18 & 0.000003485728733 & 58 & 0.001243674534966 & 98 & 0.011252349951643 \\
\hline 19 & 0.000004458431662 & 59 & 0.001357386862351 & 99 & 0.011539247717751 \\
\hline 20 & 0.000005641528829 & 60 & 0.001478613946926 & 100 & 0.011817835652354 \\
\hline 21 & 0.000007070579820 & 61 & 0.001607580745423 & 101 & 0.012087224781598 \\
\hline 22 & 0.000008786054836 & 62 & 0.001744495789436 & 102 & 0.012346541276202 \\
\hline 23 & 0.000010833800347 & 63 & 0.001889549322765 & 103 & 0.012594931134531 \\
\hline 24 & 0.000013265504756 & 64 & 0.002042911438779 & 104 & 0.012831564865668 \\
\hline 25 & 0.000016139164059 & 65 & 0.002204730201894 & 105 & 0.013055642172483 \\
\hline 26 & 0.000019519547509 & 66 & 0.002375129769048 & 106 & 0.013266396634704 \\
\hline 27 & 0.000023478663272 & 67 & 0.002554208511190 & 107 & 0.013463100391990 \\
\hline 28 & 0.000028096224097 & 68 & 0.002742037134753 & 108 & 0.013645068827001 \\
\hline 29 & 0.000033460112966 & 69 & 0.002938656803136 & 109 & 0.013811665248464 \\
\hline 30 & 0.000039666848765 & 70 & 0.003144077258185 & 110 & 0.013962305574255 \\
\hline 31 & 0.000046822051941 & 71 & 0.003358274941671 & 111 & 0.014096463014456 \\
\hline 32 & 0.000055040910163 & 72 & 0.003581191116775 & 112 & 0.014213672754439 \\
\hline 33 & 0.000064448183614 & 73 & 0.003812729989559 & 113 & 0.014313531954857 \\
\hline 34 & 0.000075178210285 & 74 & 0.004052756830457 & 114 & 0.014395699751648 \\
\hline 35 & 0.000087374911263 & 75 & 0.004301096095746 & 115 & 0.014459897256033 \\
\hline 36 & 0.000101191796024 & 76 & 0.004557529549030 & 116 & 0.014505907554518 \\
\hline 37 & 0.000116791967726 & 77 & 0.004821794382720 & 117 & 0.014533575708893 \\
\hline 38 & 0.000134348128499 & 78 & 0.005093581339514 & 118 & 0.014542808756232 \\
\hline 39 & 0.000154042584737 & 79 & 0.005372532833876 & & \\
\hline 40 & 0.000176067252389 & 80 & 0.005658241073516 & & \\
\hline
\end{tabular}

\section{Conclusion}

In this paper we have presented the mathematical demonstration that the application of Chebyshev sharpening to cosine filters results in filters with zeros on the unit circle, that is, with Minimum Phase (MP) characteristic. From this, we have proven that filters composed by a cascade of Chebyshev-Sharpened Cosine Filters (CSCFs) expanded by different factors, called cascaded expanded CSCFs, also have MP property. The cascaded expanded CSCFs are useful prefilters that provide the attenuation in an overall MP FIR filter. Moreover, these filters are a general case where the cascaded expanded cosine filters are a subset. The CSCFs are low-complexity filters, since they do not need multipliers.

It has been shown with three examples that, for a desired attenuation in the magnitude response, cascaded expanded CSCFs achieve a lower group delay in comparison to cascaded expanded cosine filters. This lower group delay is desirable in the design of MP FIR filters. Since the purpose of this paper is to prove the suitability of cascaded expanded 


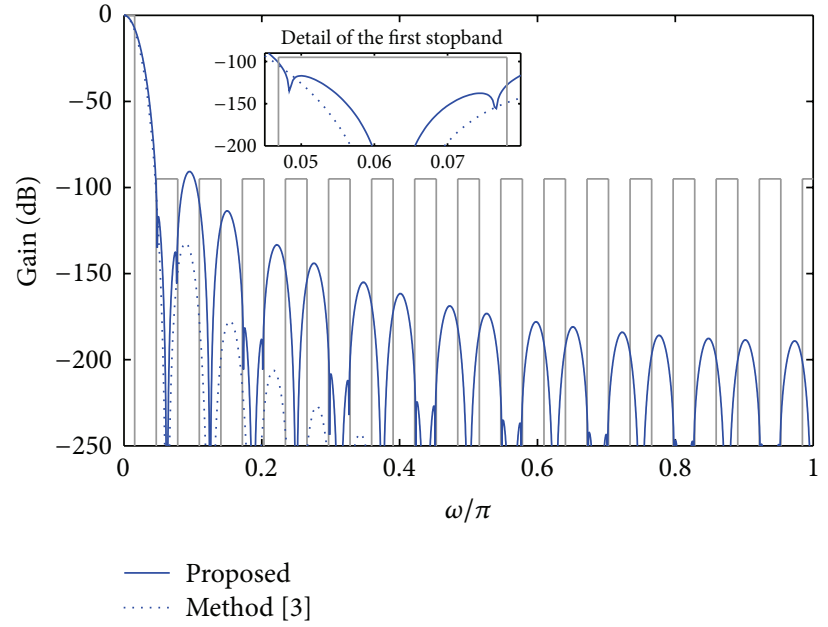

(a)

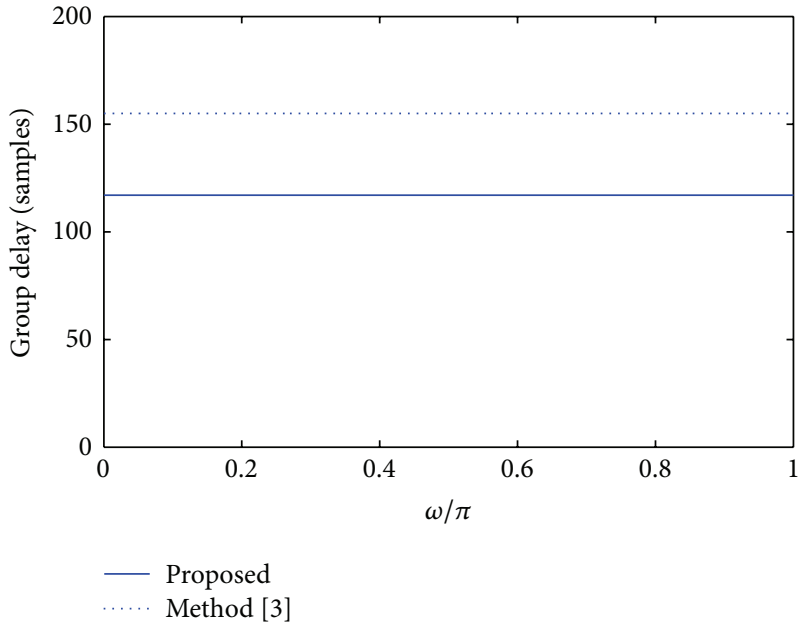

(b)

Figure 9: Magnitude responses (a) and group delays (b) of the proposed decimation filter and the filter from [3] (31), accomplishing the attenuation required in Example 3.

TABLE 8: Comparison of results in Example 3.

\begin{tabular}{lcc}
\hline & Proposed & Method [3] \\
\hline $\begin{array}{l}\text { Group delay (samples) referred to high } \\
\text { rate }\end{array}$ & 117 & 155 \\
\hline $\begin{array}{l}\text { Complexity of implementation } \\
\text { (number of adders/number of delays) }\end{array}$ & $20 / 16$ & $20 / 20$ \\
\hline $\begin{array}{l}\text { Computational complexity } \\
\text { (APOS) }\end{array}$ & 215 & 330 \\
\hline $\begin{array}{l}\text { \% improvement in group delay } \\
\text { (compared with method [3]) }\end{array}$ & $\approx 24 \%$ & - \\
\hline $\begin{array}{l}\text { \% saving in APOS } \\
\text { (compared with method [3]) }\end{array}$ & $\approx 35 \%$ & - \\
\hline $\begin{array}{l}\text { \% saving in complexity of } \\
\text { implementation } \\
\text { (compared with method [3]) }\end{array}$ & $\approx 10 \%$ & - \\
\hline
\end{tabular}

CSCFs as MP prefilters, the CSCF-based solutions provided in the examples of this work are suboptimal.

\section{Conflict of Interests}

The authors declare that there is no conflict of interests regarding the publication of this paper.

\section{References}

[1] A. V. Oppenheim and R. W. Schafer, Discrete-Time Signal Processing, Prentice Hall International, Upper Saddle River, NJ, USA, 1989.

[2] E. Hänsler and G. Schmidt, Speech and Audio Processing in Adverse Environments, Springer, 2008.

[3] Y. Wang and J. Reiss, "Time domain performance of decimation filter architectures for high resolution sigma delta analogue to digital conversion," in Proceedings of the 132nd Audio Engineering Society Convention, pp. 294-305, Budapest, Hungary, April 2012.
[4] B. Du, X. Xu, and X. Dai, "Minimum-phase fir precoder design for multicasting over MIMO frequency-selective channels," Journal of Electronics, vol. 30, no. 4, pp. 319-327, 2013.

[5] L. Aksoy, P. Flores, and J. Monteiro, "A tutorial on multiplierless design of FIR filters: algorithms and architectures," Circuits, Systems, and Signal Processing, vol. 33, no. 6, pp. 1689-1719, 2014.

[6] G. J. Dolecek and V. Dolecek, "Application of Rouche's theorem for MP filter design," Applied Mathematics and Computation, vol. 211, no. 2, pp. 329-335, 2009.

[7] J. F. Kaiser and R. W. Hamming, "Sharpening the response of a symmetric nonrecursive filter by multiple use of the same filter," IEEE Transactions on Acoustics, Speech, and Signal Processing, vol. 25, no. 5, pp. 415-422, 1977.

[8] J. O. Coleman, "Chebyshev stopbands for CIC decimation filters and CIC-implemented array tapers in 1D and 2D," IEEE Transactions on Circuits and Systems I: Regular Papers, vol. 59, no. 12, pp. 2956-2968, 2012.

[9] M. O. Rayes, V. Trevisan, and P. S. Wang, "Factorization properties of Chebyshev polynomials," Computers \& Mathematics with Applications, vol. 50, no. 8-9, pp. 1231-1240, 2005.

[10] H. J. Oh and Y. H. Lee, "Design of discrete coefficient FIR and IIR digital filters with prefilter-equalizer structure using linear programming," IEEE Transactions on Circuits and Systems II: Analog and Digital Signal Processing, vol. 47, no. 6, pp. 562-565, 2000.

[11] A. Fernandez-Vazquez and G. J. Dolecek, "Passband and stopband CIC improvement based on efficient IIR filter structure," in Proceedings of the 53rd IEEE International Midwest Symposium on Circuits and Systems (MWSCAS '10), pp. 765-768, August 2010.

[12] T. Saramaki, "Finite impulse response filter design," in Handbook for Digital Signal Processing, S. K. Mitra and J. F. Kaiser, Eds., pp. 155-277, John Wiley \& Sons, New York, NY, USA, 1993.

[13] D. E. T. Romero and G. J. Dolecek, "Application of amplitude transformation for compensation of comb decimation filters," Electronics Letters, vol. 49, no. 16, pp. 985-987, 2013.

[14] G. J. Dolecek and A. Fernandez-Vazquez, "Trigonometrical approach to design a simple wideband comb compensator," 
International Journal of Electronics and Communications, vol. 68, no. 5, pp. 437-441, 2014.

[15] S.-C. Pei and S.-T. Lu, "Design of minimum-phase FIR filters by differential cepstrum," IEEE Transactions on Circuits and Systems, vol. 33, no. 5, pp. 570-576, 1986.

[16] H. H. Dam, S. Nordebo, and L. Svensson, "Design of minimumphase digital filters as the sum of two allpass functions using the cepstrum technique," IEEE Transactions on Signal Processing, vol. 51, no. 3, pp. 726-731, 2003.

[17] S.-C. Pei and H.-S. Lin, "Minimum-phase FIR filter design using real cepstrum," IEEE Transactions on Circuits and Systems II: Express Briefs, vol. 53, no. 10, pp. 1113-1117, 2006.

[18] O. Herrmann and H. W. Schüessler, "Design of nonrecursive digital filters with minimum-phase," Electronics Letters, vol. 6, no. 11, pp. 329-330, 1970.

[19] I. Kale, G. D. Cain, and R. C. S. Morling, "Minimum-phase filter design from linear-phase startpoint via balanced model truncation," IET Electronic Letters, vol. 31, no. 20, pp. 1728-1729, 1995.

[20] T. Stathaki and I. Fotinopoulos, "Equiripple minimum phase FIR filter design from linear phase systems using root moments," IEEE Transactions on Circuits and Systems II: Analog and Digital Signal Processing, vol. 48, no. 6, pp. 580-587, 2001.

[21] M. Okuda, I. R. Khan, M. Ikehara, and S. Takahashi, "Quasiequiripple approximation of minimum phase FIR filters by updating desired response," IEE Proceedings: Vision, Image and Signal Processing, vol. 151, no. 3, pp. 164-169, 2004.

[22] M. Okuda, M. Ikehara, and S.-I. Takahashi, "Design of equiripple minimum phase FIR filters with ripple ratio control," IEICE Transactions on Fundamentals of Electronics, Communications and Computer Sciences, vol. 89, no. 3, pp. 751-756, 2006.

[23] E. B. Hogenauer, "An economical class of digital filters for decimation and interpolation," IEEE Transactions on Acoustics, Speech, and Signal Processing, vol. 29, no. 2, pp. 155-162, 1981. 


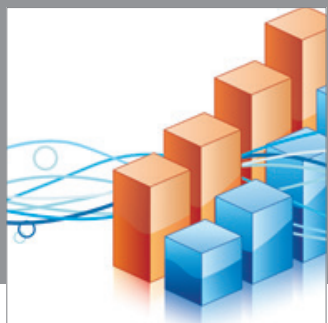

Advances in

Operations Research

mansans

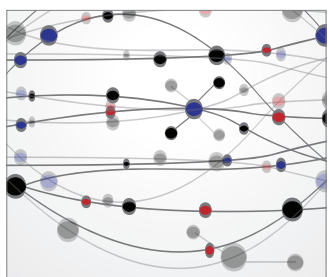

The Scientific World Journal
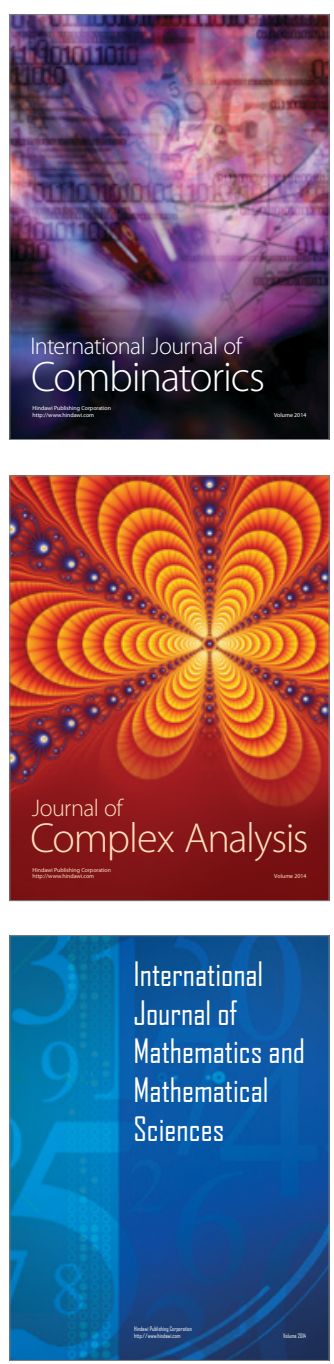
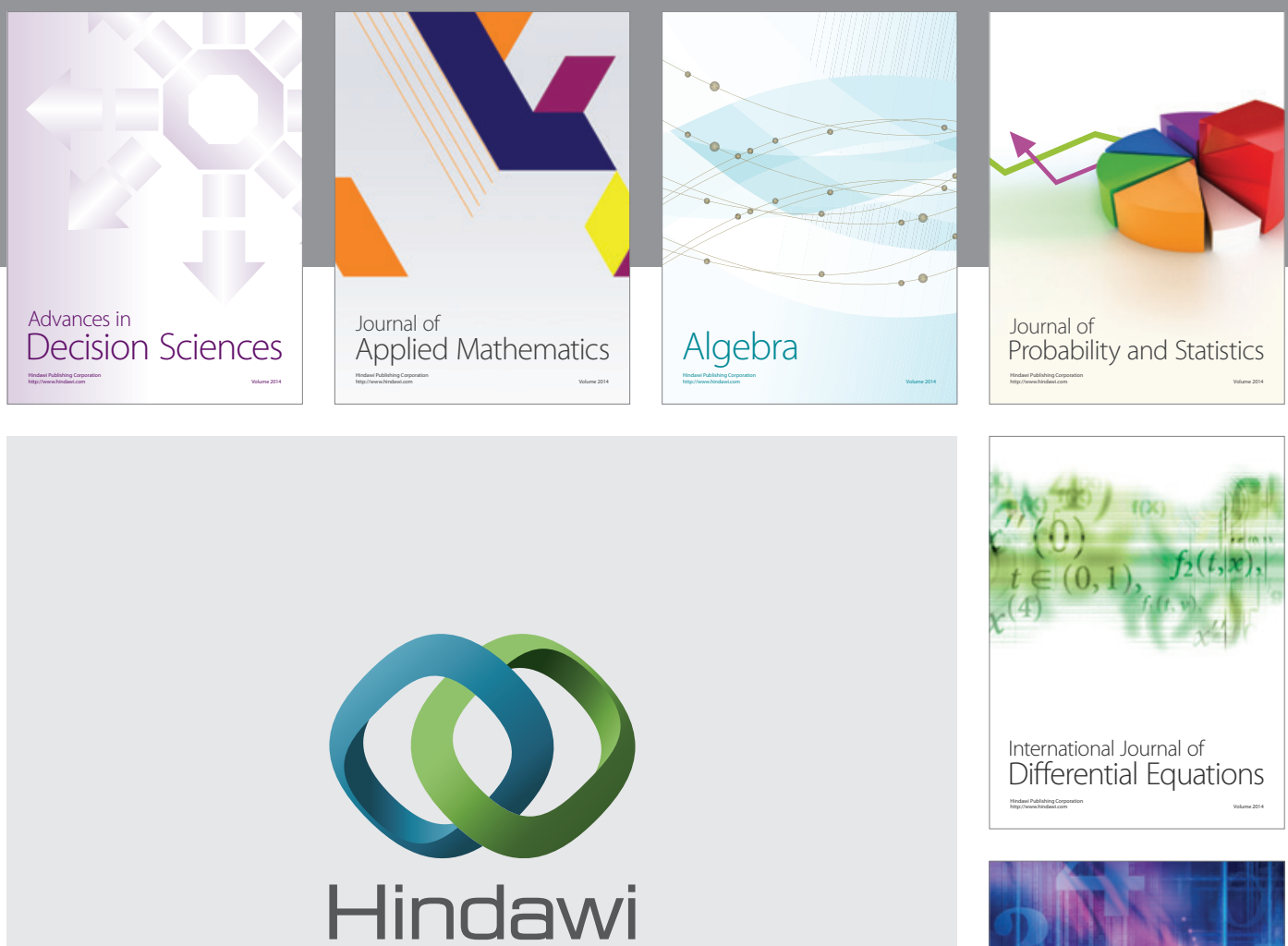

Submit your manuscripts at http://www.hindawi.com
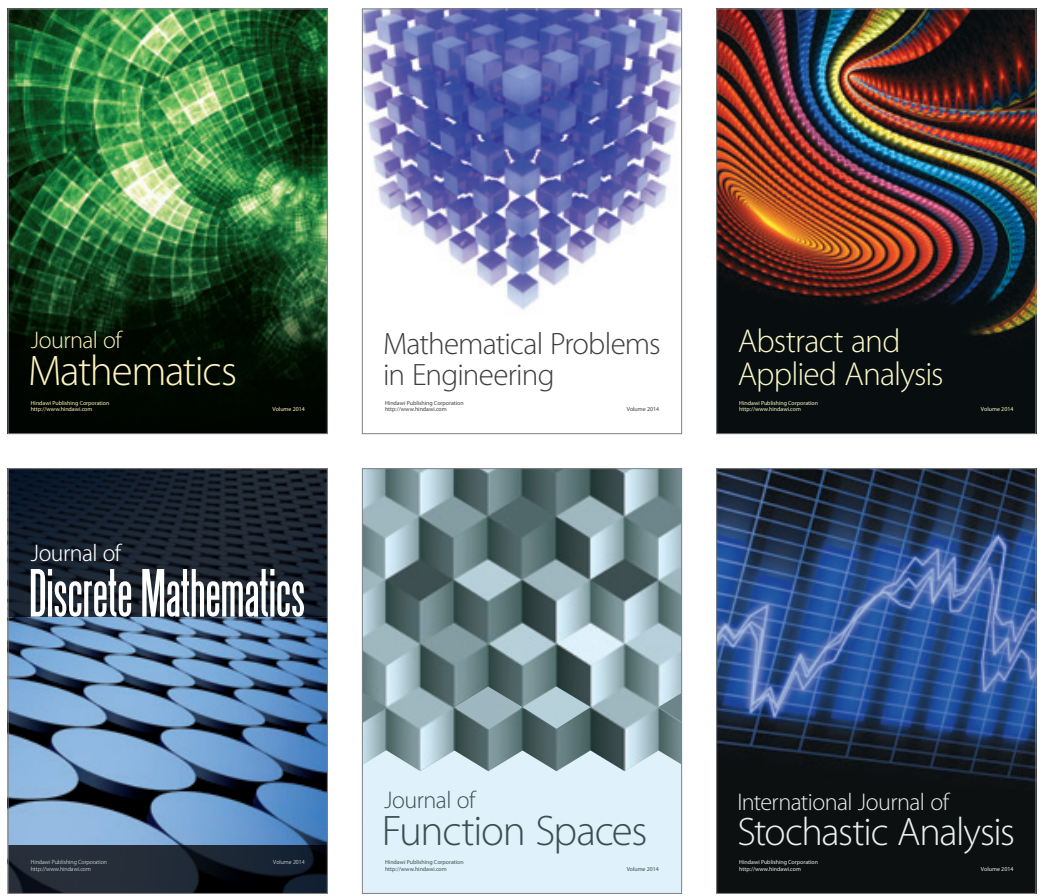

Journal of

Function Spaces

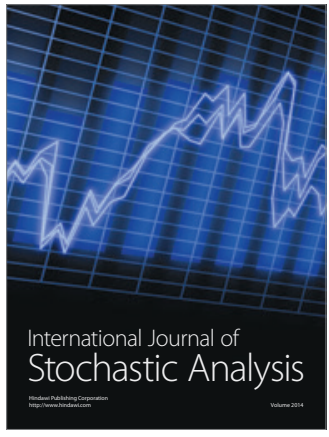

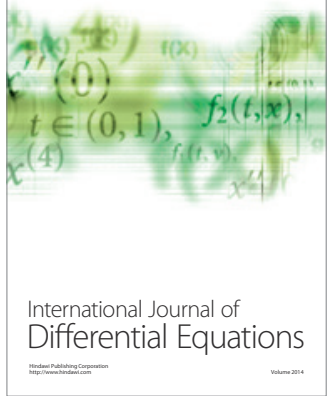
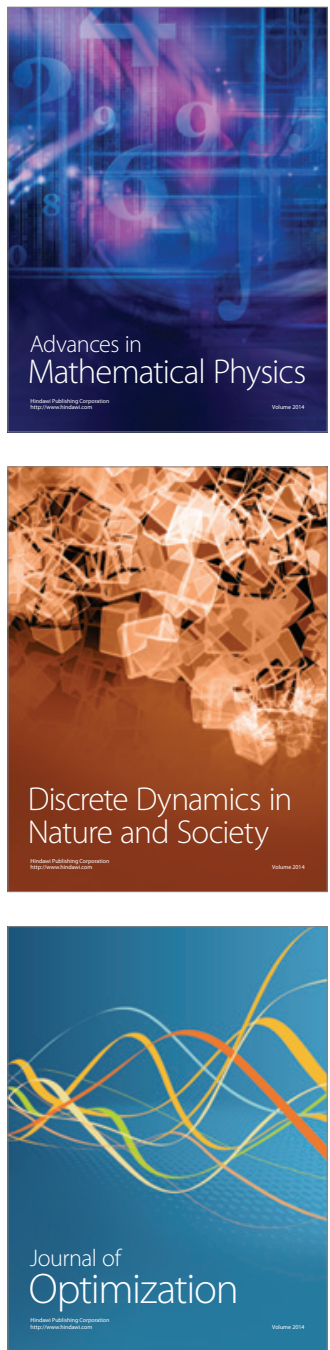